\title{
OS DESAFIOS DA FORMAÇÃO DE PROFESSORES DE MATEMÁTICA PARA O USO DE TECNOLOGIAS COMPUTACIONAIS SOB A TEORIA HISTÓRICO- CULTURAL
}

\section{THE CHALLENGES OF TRAINING OF MATHEMATICS TEACHERS FOR THE USE OF COMPUTATIONAL TECHNOLOGIES UNDER HISTORICAL- CULTURAL THEORY}

\section{LOS RETOS A LA FORMACIÓN DE PROFESORES DE MATEMÁTICA PARA EL USO DE LAS TECNOLOGÍAS DE COMPUTACIÓN DESDE LA PERSPECTIVA DE LA TEORÍA HISTÓRICO CULTURAL.}

\author{
Francielle de Mattos \\ Graduação em Matemática - UFSCar \\ Mestrado em Ciências da Computação - UFSCar \\ Doutoranda em Educação - UFSCar \\ Email: mattosfrancielle234@gmail.com \\ Priscila de Mattos \\ Graduação em Matemática - USP São Carlos \\ Mestrado em Educação - FFCLRP - USP \\ E-mail: priscilademattos86@gmail.com
}

\section{RESUMO}

O presente artigo discute os desafios da formação de professores de matemática para o uso de tecnologias computacionais, assumindo a Teoria Histórico-Cultural como referencial teórico. Para tal, integramos o contexto tecnológico com a formação inicial e continuada de professores de matemática. Com isso, é possível prover instrumentos para que o docente vivencie em sala de aula o processo de ensino e aprendizagem, em que haja apropriação dos conceitos científicos matemáticos. Assim, apoiado pela compreensão dos conceitos historicamente desenvolvidos pela humanidade, o docente pode tomar suas decisões de forma mais consciente ao organizar o ensino como atividade. Tais decisões são ações permanentes de quem está em atividade de ensino, nesse caso, a matemática. Portanto, ao organizar os processos de ensino e de aprendizagens sob um novo método surge a preocupação de que este contemple o trabalho pedagógico emanado dos conhecimentos teóricos. Na intenção de sistematizar a cultura humana e torná-la acessível aos estudantes, este artigo propõe uma seja revisto a forma de inserção das tecnologias como recurso na atividade pedagógica, não apenas como finalidade e um meio em si, mas que promova a aprendizagem.

Palavras-chave: educação matemática. Formação de professores. Tecnologia na educação. Teoria Histórico-Cultural.

Palavras-chave: educação matemática. Formação de professores. Tecnologia na educação. Teoria Histórico-Cultural.

\section{ABSTRACT}

The present article tries to explode the challenges of the formation of teachers of mathematics for the use of computational technologies, assuming the historical-cultural theory as 
theoretical reference. It integrates the technological context with the initial and continuous training of mathematics teachers. Provide instruments so that the teacher can experience in the classroom moments of (re) signification of mathematical concepts and pedagogical practice. The development of the understanding of concepts historically developed by humanity allows the teacher to become aware of the time to organize teaching as an activity, permanent action of those who are in the teaching activity, specifically mathematics. When organizing the teaching-learning process under a new method, the concern is that it should contemplate the pedagogical work that emanates from theoretical knowledge. To systematize human culture and make it accessible to students. Therefore, the article proposes a look of overcoming the challenges of the insertion of technologies as a resource of pedagogical activity.

Keywords: Mathematics education. Teacher formation. Technology in the education. Cultural- Historical Theory,

\section{RESUMEN}

El presente artículo aborda los retos de la formación de profesores de matemáticas para el uso de las tecnologías de computación, adoptando como referente la Teoría Histórico-Cultural teórico. Así, integramos el contexto tecnológico con la formación inicial y continuada de profesores de matemáticas y proponemos instrumentos para que el docente vivencie un proceso de enseñanza y aprendizaje, en el que haya apropiación de los conceptos científicos matemáticos. El desarrollo de la comprensión de los conceptos históricamente desarrollados por la humanidad permite al docente la toma de conciencia en el momento de organizar la enseñanza como actividad, acción permanente de quien enseña, en este caso, las matemáticas. Por lo tanto, al organizar los procesos de enseñanza y de aprendizajes bajo un nuevo método surge la preocupación de que éste contemple el trabajo pedagógico que emana de los conocimientos teóricos, con la intención de sistematizar la cultura humana y hacerla accesible a los estudiantes. Consecuentemente, el artículo propone una revisión de la forma en que ocurre la inserción de las tecnologías como recurso en la actividad pedagógica, no sólo como finalidad y un medio en sí, con el objetivo de que promueva el aprendizaje.

Palabras clave: Educación Matemática, Formación de Profesores, Tecnología Educativa, Teoría Histórico-Cultural.

\section{INTRODUÇÃO}

Este artigo consiste em um estudo sobre as relações entre a educação escolar e o desenvolvimento das funções psíquicas superiores a partir da unidade dialética entre o ensino e a aprendizagem, ou seja, a atividade pedagógica. Trazendo os principais desafios da utilização de instrumentos pedagógicos fortalecedores do aspecto metodológico na atividade de pedagógica, sob a Teoria Histórico-Cultural.

Consideramos que a unidade dialética se estabelece quando, em atividades particulares, estudantes e professores se integram em uma atividade em comum, a qual é movida por motivos, necessidades, objetivos, ações e operações que correspondem entre si. Assim, ações e operações na atividade de ensino e na atividade de estudo tem como 
objetivo comum o desenvolvimento humano por meio da apropriação da produção cultural humana.

Logo, o objetivo desse estudo é de possibilitar que o docente em sala de aula reconheça a necessidade de propor atividades pedagógicas, reconhecendo a zona de desenvolvimento proximal dos estudantes, utilizando os instrumentos computacionais tecnológicos. E que o trabalho docente, pautado no conceito de mediação, estabeleça relação entre o estudante a ferramenta e, assim, possibilite a superação e atue na zona de desenvolvimento próximal. E que vise o desenvolvimento das funções psicológicas superiores, tais como: imaginação, atenção voluntária, memória, percepção, domínio da conduta, formação de conceitos e o raciocínio lógico-matemático (VYGOTSKY, 1995).

Partimos então, dos estudos de Vygotski (1995, 2001), Leontiev (1978, 1983), Davýdov (1982,1988), em particular a constituição de desenvolvimento das funções psicológicas superiores, e sobre as possibilidades de organização do ensino que possibilitem condições favoráveis para que ocorra a apropriação dos conceitos científicos nos espaços escolares. Também buscamos embasamento em estudos decorrentes dos mencionados anteriormente, como os de Moura; Sforni; Araujo (2011), que tratam da atividade de ensino e de aprendizagem como aquela que está emanada do conhecimento históricamente elaborado, no caso específico os matemáticos. Além, de outros autores que estudam o uso de recursos tecnológicos computacionais no ensino de matemática e de autores que se dedicam ao estudo de formação de professores.

Ao tratarmos do ensino da Matemática e do cenário atual da sociedade, e da necessidade do uso de novos instrumentos tecnológicos de forma que esteja alinhado com as novas Tecnologias de Informação e Comunicação (TIC), há de fazer sentindo na organização do ensino e na formação dos professores. No entanto, o ensino de matemática ainda se concentra no formalismo lógico, na memorização de fórmulas e no conteúdo em si, como era no século XX (MARCO, 2009). Nessa lógica formal, o aluno precisa decorar fórmulas e aplicá-las na resolução de exercícios matemáticos, mesmo sem a devida compreensão de quando e por que realmente utilizá-las.

Nesse cenário é preciso relacionar as fórmulas e os conteúdos com o processo de produção científica, valorizando o conhecimento como produções culturais e de desenvolvimento do ser humano (VYGOTSKY, 1995). Além disso, a mecanização do ensino e a deficiência na compreensão dos conceitos ensinados, relacionando-os com o 
contexto real de aplicação, têm contribuído para o distanciamento dos indivíduos de certos conceitos, mais complexos, de matemática (LIMA, 1998).

$\mathrm{Na}$ proposta de Davýdov, os professores em atividade pedagógica organizam intencionalmente o ensino em forma de atividade de estudo e/ou ações de aprendizagem, por meio das sínteses e abstrações que revelam as relações essenciais do(s) fenômeno(s). A atividade pedagógica tem como objeto a transformação, conforme a concepção de revolução, dos sujeitos e é por meio dessa atividade (teórico-prática) que se faz necessária a apropriação da produção cultural humana.

Ir para além da aparência caracteriza a apropriação do conhecimento científico que possibilita generalizações; não as genéricas, mas aquelas que estão vinculadas ao pensamento teórico. Ou seja, é pela organização do ensino voltado à formação do pensamento teórico nos estudantes que se faz possível a apropriação dos conceitos propriamente ditos dos números reais.

Davídov (1988) destaca que no processo de ensino-aprendizagem o desenvolvimento psicológico é aquele que promove o sujeito a estar em atividade. Assim, a criança, além do conhecimento científico, desenvolve as capacidades construídas historicamente, que permeiam a formação da consciência e pensamento teórico. Essas capacidades de origem histórica são: a reflexão, a análise e a experiência mental.

Nesse sentido, Leontiev (1978) fala que todo homem nasce como potencial candidato a ser humano, e que somente se constrói humano quando se apropria da cultura produzida historicamente pela atividade. É na relação com os objetos, mediada pelas relações humanas, que a criança terá a possibilidade de se humanizar, a essa atividade na “infância” Leontiev denominou educação. A esse sujeito em sua

especificidade histórica é permitida a apropriação do estágio de desenvolvimento humano no seu grau mais elevado, como dito anteriormente, sem que haja necessidade de reinventar os conceitos a cada nova geração, podendo, assim, superar tal estágio.

Para Vigotski (2007), o sujeito, ao se apropriar dos conceitos científicos, precisa não apenas ser capaz de entender o aspecto empírico e observável dos fenômenos. $\mathrm{O}$ conceito científico só se faz internalizado pelo movimento do pensamento, da consciência, envolvendo funções psicológicas superiores, não apenas a memorização. É preciso mudar, segundo Davýdov (1982), a concepção sobre o ensino dos conceitos matemáticos, pois há uma hipervalorização dos processos de repetição e memorização. 
Segundo Davýdov (1988), o pensamento empírico realmente dificulta o desenvolvimento do pensamento teórico, pois generalizar situações particulares leva a abstrações empíricas. Mas ressalva que:

[...] de nenhuma maneira, menosprezamos o papel da memória. Mas é indispensável empregar métodos que favoreçam o desenvolvimento mental. A prática de lembrar sem compreender influencia negativamente na formação de entendimentos independentes, dificulta o desenvolvimento do pensamento crítico e racional (DAVÍDOV, 1988, p. 163).

Portanto, o uso de tecnologias computacionais no processo de ensino e aprendizagem tem sido amplamente discutido na comunidade de educadores, em especial os matemáticos. Tomando com a Teoria Histórico-Cultural, o método dialético nos levou a considerar que o manuseio dos recursos tecnológicos em aula por si só não leva a aprendizagem dos conceitos. Dentre essas discussões, destacamos a compreensão de como o professor de matemática pode utilizar esses recursos tecnológicos em conjunto com a produção de situações e atividades. De forma que possam desenvolver o pensamento teórico, em relação aos conceitos matemáticos.

Dessa forma, esses recursos visam envolver o estudante no processo de aprendizagem, permitindo que ele dê novos sentidos e, aproprie-se dos conceitos matemáticos, de acordo com a Teoria da Atividade proposta por Leontiev (1978). Além disso, consideramos tal teoria para analisar os sentidos, como soma de todos os acontecimentos psicológicos que uma palavra/conceito desperta na consciência a partir de um contexto específico (VYGOTSKY,1995), atribuídos pelos sujeitos à organização do ensino.

Portanto, os recursos computacionais educacionais auxiliam o docente no momento da organização do ensino com vistas ao desenvolvimento do pensamento teórico humano, possibilitando a interpretação e reflexão do contexto, resolução de problemas e produção de atividades (LANNER DE MOURA, MISKULIN E MELO, 2000).

Valente e Almeida (1997) destacam a importância da informática na educação e da sua aceitação pelos educadores para consolidá-la no sistema educacional. Tal fato é um dos obstáculos a serem ultrapassados para aumentar a qualidade do processo de ensino e aprendizagem, alinhando-o com as constantes inovações. E assim, permitir a 
apropriação das máximas produções humanas nas ações pedagógicas e, compreender o movimento do pensamento teórico, ou seja, o surgimento e desenvolvimento dos conceitos (DAVÝDOV, 1982; DIAS, 2013; GIARDINETTO, 1999; ROSA, 2012).

\section{A FORMAÇÃO INICIAL E CONTINUADA DE PROFESSORES DE MATEMÁTICA PARA O USO DE TECNOLOGIA}

A formação de professores tem sido abordada em inúmeras pesquisas nacionais e internacionais. Dentre os pesquisadores nacionais que discutem formação de professores que ensinam matemática destacam-se as pesquisas de Beatriz D'Ambrósio (1993), Fiorentini e Castro (2003), Araújo (2003), Moura (2004), Freitas (2006), entre outros.

A formação inicial e continuada de professores é indispensável para a melhoria da qualidade da educação básica, sendo considerada um componente de extrema relevância nas políticas públicas brasileiras (MELLO, 2000), porém nem sempre colocada de maneira a trazer a libertação e não alienação como a política das competências (MORETTI; MOURA, 2010).

Assim como educação, ensino, e outros, formação envolve, necessariamente, uma dimensão interpsíquica, no plano social, depois intrapsíquica no plano psicológico de desenvolvimento cultural humano (VYGOTSKI, 1995).

Nesse contexto, percebe-se que o fenômeno "formação de professores", apesar de ser um campo com vasta discussão na academia, apontando para diferentes compreensões sobre o tema, ainda merece especial atenção, considerando "a complexidade do objeto principal do professor: o ensino" (MOURA, 2004).

O docente em atividade de organização do ensino, ou seja, em atividade pedagógica, está diante de um movimento em que precisa colocar o estudante em situação de ensino e de posse dos instrumentos mais sofisticados para assim, apropriar-se dos signos (conceitos) em suas máximas potencialidades (VYGOTSKY, 1995).

Uma importante ação dentro dessa atividade de estudo é apontar para a mobilização de políticas de formação de professores, já queimplicam a articulação entre formação inicial e formação continuada, e que as políticas públicas e instituições envolvidas devam atuar de modo articulado nos diferentes níveis e modalidades da educação básica (MARCO, 2009). 
A utilização de tecnologias computacionais na formação inicial de professores de matemática é de fundamental importância, pois se concretiza na necessidade de a universidade estar em sintonia com os licenciandos, que na maioria são jovens imersos nesse campo tecnológico (BONILLA, 2002). Logo, é papel da escola promover a apropriação do conhecimento científico, por isso a realidade é elemento essencial do processo de ensino aprendizagem (DAVÝDOV, 1982).

O desenvolvimento dos conceitos científicos, surge de uma situação mediada intencionalmente em que se busca explorar a zona de desenvolvimento proximal, logo o que está em processo de apropriação e objetivação (VYGOTSKY, 1995). Além de compreender os processos de desenvolvimento pessoal e profissional do professor, é necessário considerá-lo detentor de uma profissão na qual o próprio sujeito histórico é capaz de produzir o seu próprio ofício. Portanto, os conceitos cotidianos se diferem dos científicos por se desenvolverem "de baixo para cima", ou seja, da experiência cotidiana - concreta/particular - para a generalização (VYGOTSKI, 2005). Os conceitos científicos imprimem um movimento "de cima para baixo", ao partir das generalizações para o particular (VYGOTSKI, 1995).

\section{TECNOLOGIAS COMPUTACIONAIS NO ENSINO DE MATEMÁTICA}

O inegável avanço técnico-científico propiciado pelas tecnologias computacionais tem levado aos pesquisadores e profissionais de educação a refletirem sobre os processos de ensino e de aprendizagem, fazendo o uso desses recursos, envolvendo o professor, o aluno e o saber, nesse caso, matemático.

Sobre o uso de TICs na educação, é preciso ressaltar que elas podem ser consideradas inimigas ou aliadas para a prática pedagógica, de acordo com o ponto de vista adotado. Elas podem ser inimigas quando sua incorporação segue apenas aspectos econômicos das comunicações ou quando só contribuem para a diversão sem promover o conhecimento em situação mediada. Entretanto, elas podem ser aliadas quando tornam acessíveis informações de qualidade, permitindo a pesquisa, a criação e a interação com os conteúdos (LESSARD \& TARDIF, 2008).

Pesquisadores, como Papert (1994), Valente e Almeida (1997), Valente (1998), Azinian (1998), Miskulin, Lanner de Moura e Silva (2003), Borba e Penteado (2003), 
demonstram a importância dos recursos computacionais no contexto educacional, indicando que a educação tem função social e emancipadora. Além disso, a educação deve proporcionar aos indivíduos acesso aos saberes e às formas culturais inerentes ao contexto social a que pertencem, é ter acesso à riqueza humana produzida, promovendo desenvolvimento e aprendizagem.

A disseminação e a utilização de computadores nas escolas precisam estar consolidadas no sistema educacional para poder propiciar uma melhor qualidade de ensino. No entanto, Campos (2007) relata alguns problemas recorrentes à adoção não planejada de TICs no sistema educacional. Ele verificou que em várias escolas os laboratórios de informática são pequenos e possuem poucas máquinas para atender os estudantes. Faltam ainda profissionais, técnicos e professores com formação adequada para utilizá-las como recursos auxiliares na atividade pedagógica e de ensino.

Recursos computacionais e tecnológicos podem ser utilizados no ensino de matemática como ferramentas de auxílio à aprendizagem, proporcionando ao aluno um ambiente de aprendizagem que oferece condições para fazer simulações que seriam difíceis ou impossíveis de serem realizadas por meio de lápis e papel (RODRIGUES, SOUZA JÚNIOR E LOPES, 2007). Dessa forma, a mídia digital pode favorecer a verificação de hipóteses e conjecturas levantadas pelos alunos de maneira mais dinâmica e interativa. Com isso, as tecnologias computacionais podem auxiliar o professor na elaboração de atividades de ensino que permitam ao aluno estar em situação investigativa, no qual eles podem representar os conhecimentos adquiridos e, até mesmo (atribuí-los novos significados (MISKULIN, LANNER DE MOURA E SILVA, 2003; VYGOTSKY, 1995).

Niess et al. (2009) mencionam que as TICs não devem ser utilizados apenas como ferramentas para apresentação e verificação de ideias previamente estabelecidas sem o uso da tecnologia e para realizar atividades do tipo repetição e prática, conhecidas pelo termo em inglês drill and practice. Com isso, para os professores, ensinar com TICs ainda é um desafio, uma vez que eles precisam incorporar o uso dessas tecnologias para promover aprendizagem em termos da matemática. De forma que, aprendizagem seja concebida enquanto experiência social e mediada pela interação entre a linguagem e ação. Nessa perspectiva, o recurso tecnológico não deve ser utilizado apenas para fins ilustrativos ou de apoio à aula, pois estará apenas reproduzindo os métodos de ensino 
considerados tradicionais.

Planejar uma aula com recursos tecnológicos exige do professor uma fundamentação teórica e conhecimento dos recursos disponíveis pelas tecnologias empregadas. Dessa forma, a efetiva contribuição dos recursos tecnológicos nos processos de ensinos e de-aprendizagens está diretamente relacionada aos recursos disponíveis nas ferramentas e o modo como eles são utilizados (GRAVINA \& SANTAROSA, 1998). Uma aula dinâmica, criativa e segura por meio do uso de TICs somente é possível se o professor escolher bem e conhecer profundamente as ferramentas escolhidas para as atividades de ensino (TAJRA, 2001), é o planejamento das ações de ensino e estudo. Outro desafio para os professores é a compreensão em relação a quando ou em que etapa do processo de ensino e aprendizagem o recurso tecnológico deve ser inserido.

A utilização de computadores no ensino de matemática pode desencadear uma nova relação professor-aluno, marcada por uma maior proximidade, interação e colaboração, quando utilizada de forma intencional e com objetivos claros. Tal fato exige uma nova concepção e formação de professor. Um recurso bastante explorado por algumas universidades públicas e particulares é a criação de Objetos de Aprendizagem $(\mathrm{OA})$

Wiley (2001) define que um objeto de aprendizagem consiste em qualquer recurso digital que possa ser reutilizado como apoio ao ensino, com uma ideia central em que o conteúdo educacional seja reaproveitado em diferentes contextos e ambientes, o que não torna rígido a sua utilização. Os OAs podem ser integrados ao currículo dos ensinos fundamental, médio e até mesmo superior. Dentre os principais desafios para os OAs incluem, a falta de divulgação dos repositórios existentes e de OAs que se adequem às demandas pedagógicas dos professores.

A partir dessa premissa, o processo de formação de funções psíquicas superiores acontecerá mediante as condições oferecidas pelo docente, na sua atividade pedagógica, na organização do currículo e ensino, os quais exigem solidez na teórica aqui adotada. Para que o sujeito possa tomar consciência e ter autocontrole, princípios e práticas convergentes são elementos importantes no princípio da atividade humana e potencializadores de aprendizagem que desencadeiam novas estruturas superiores (VYGOTSKY, 1995). 


\section{USO DE TECNOLOGIAS COMPUTACIONAIS SOB A TEORIA HISTÓRICO CULTURAL NA FORMAÇÃO DO PROFESSOR DE MATEMÁTICA}

Primeiramente, evidencia-se que os pesquisadores da Educação Matemática que se fundamentam na Teoria Histórico-Cultural recorrem às pesquisas e categorias propostas por Vygotsky (1995), sobre o desenvolvimento intelectual humano. Em suas obras, Vygotsky emanado pelas ideias marxistas e compreende o papel central das interações sociais e condições de vida do sujeito para o seu desenvolvimento intelectual (LEANDRO, 2017). Com isso, o homem transforma o ambiente e utiliza conhecimentos anteriormente adquiridos em sua relação com o meio, em um processo histórico e cultural (OLIVEIRA, 1997).

Borges (2000), se vale de Vygotsky, ao defender que as intervenções em sala de aula ocorrem pelos signos e por outros instrumentos culturais produzidos historicamente pela sociedade humana em suas relações de trabalho. Dessa forma, não basta o simples manuseio de instrumentos para que o ser humano aprenda, é preciso que o indivíduo entenda as origens e o desenvolvimento teórico para que ocorra a compreensão desse conceito. A cultura é um produto do trabalho humano, ela expressa o processo histórico, objetivando signos e instrumentos, podendo esses serem da forma material, ou psicológico (VYGOTSKY, 1995).

Segundo Santaella (1996), o computador é um objeto social que favorece e faz a mediação do homem com o mundo concreto, funcionando como extensão ou prolongamento de suas habilidades. Ao mesmo tempo que o homem comanda a máquina, seus conhecimentos podem se ampliar, uma vez que Borba e Penteado (2003), destacam a ideia da tecnologia computacional como uma extensão da memória do homem.

Ressaltamos que o ambiente computacional em si, por mais rico, construtivo e interativo que seja, não é autossuficiente para garantir a interiorização do conceito/ conhecimento A utilização sem planejamento intencional de ambientes computacionais nas escolas não implicará no desenvolvimento do pensamento teórico, ficando apenas, possivelmente, no pensamento empírico (DAVÝDOV, 1988).

O estudante precisa vivenciar um ensino que promova seu desenvolvimento, que o faça superrar os obstáculos no processo de conhecimento, reconhecendo que a memória concreta, a percepção direta, entre outros, não são suficientes para que ao resolver um problema e se aproprie de novos conceitos. Segundo Vygotski (1995, p. 272, tradução 
nossa) “o desenvolvimento da linguagem da criançã influencia o pensamento e o reorganiza". Ou seja, o meio visual empregado no recurso computacional deve valer-se da linguagem para organizar o pensamento do estudante sobre o objeto em estudo e, não apenas valorizar a repetição e memorização. Como muito se faz nas aulas de matemática nos laboratório de informatica, em que o professor propõe jogos de memória, de percurso, de realização de operações com preocupação apenas no algoritmo.

Assim, os meios visuais e verbais precisam combinar entre si nos recursos tecnológicos computacionais que fazem parte da organização do ensino de certo conceito e conteúdos. Em que o estudante em atividade de estudo desenvolvam a percepção e conhecimento de objetos e fenômenos, implicando no desenvolvimento das funções psíquicas.

E por isso, o professor tem um papel fundamental no processo de ensino aprendizagem, o de organizar o ensino e a atividade. Dessa forma, por meio de TICs, o professor pode propor situações desencadeadoras, aos alunos, permitindo que eles desenvolvam a criatividade, busquem novos caminhos e se envolvam no processo de aprendizagem. Nesse percurso metodológico, as TICs podem ser utilizadas como ferramentas externas, potencializando o desenvolvimento intelectual dos alunos (MARCO, 2009).

O fenômeno da formação de professores é complexo e existe pouco consenso em relação às teorias e dimensões mais relevantes para sua análise. Investigando o processo de formação dos professores de matemática desde sua formação, Marco (2009) evidenciou a necessidade de vivenciar e explorar as atividades de ensino, bem como produzir atividades de ensino em ambientes computacionais. Dessa forma, os cursos de formação de professores não devem propiciar apenas o domínio técnico de ferramentas computacionais, mas também colaborar para a construção de conhecimentos matemáticos. Esses cursos devem formar professores que tenham sensibidade para refletir sobre o modo como essas ferramentas podem contribuir para e na sala de aula (MARCO, 2009).

\section{CONCLUSÃO}

As tecnologias computacionais fazem parte da realidade da sociedade

$$
\text { RELEDUC | ISE | v. } 2 \text { | n. } 1 \text { | fev. } 2019
$$


contemporânea. Dessa forma, este artigo nos mostra os desafios propostos sobre o papel dessas tecnologias no apoio ao ensino de matemática. Tal objetivo ainda é desafiador para a área de educação, uma vez que a formação de professores deve preparar os profissionais para dominar essas tecnologias, considerando a evolução delas, sabendo dispor das mesmas ao organizar o ensino. Com isso, por mais que esta proposta esteja instanciada na formação de professores de matemática, outras áreas de licenciatura também poderão se apropriar de certos conhecimentos evidenciados e levantados durante a realização deste trabalho.

A pesquisa buscou compreender e analisar estudos sobre a importância do uso das TICs na atividade pedagógica, nas aulas de matemática, como potencializadores das funções psicológicas superiores, com base na teoria histórico-cultural, na atividade pedagógica mediada.

Com isso, elencamos alguns dos principais desafios na formação de professores de matemática para o uso de tecnologias computacionais sob a teoria histórico-cultural são: (1) a necessidade de estudos que auxiliem o professor de matemática no processo de seleção e integração de tecnologias computacionais na organização do ensinode matemática; (2) de forma intencional, considerando a sua dimensão ontológica da constituição do psiquismo e da dimensão histórica do próprio conhecimento; (3) estudos que tragam como temática a compreensão do planejamento como elemento organizador da atividade pedagógica e a significação da ação de planejar, que considere a dimensão da formação da consciência e personalidade e da apropriação da cultura humana, consequententemente no espaço escolar do conhecimento teórico; (4) alterações do currículo que convirjam para os objetivos dos cursos de graduação, em que haja a preocupação com a compreensão do papel dos instrumentos tecnolócos na atividade de ensino e assim, atendam as necessidades de integração de TICs à prática pedagógica; (5) a implementação de políticas públicas que forneçam cursos de extensão para formação continuada, uma vez que as tecnologias evoluem constantemente; (6) a criação de um fórum para promover a discussão, colaboração e troca de experiências entre professores de matemática no uso de TICs.

Além disso, as tecnologias precisam também ser amplamente disponíveis e flexíveis, adaptando-se às necessidades pedagógicas dos professores. Por fim, os professores também precisam estar receptivos para adotar essas tecnologias e apoiados 
pelas instituições das quais eles fazem parte.

\section{REFERÊNCIAS}

ALMEIDA, M. E. O computador na escola: contextualizando a formação de professores praticar a teoria, refletir a prática. (Tese de Doutorado). Programa de PósGraduação em Educação: Currículo, Pontifícia Universidade Católica, São Paulo, 2000.

AZINIAN, H. Capacitación docente para la aplicación de tecnologias de la formación en el aula de geometria. In: Congresso Ibero-Americana De Informática Na Educação, 4., 1998, BRASÍLIA, DF. Anais... . p. 1-9.

BONILLA, M. H. S. Inclusão digital e formação de professores. Revista de Educação, v. 11, p.43-50, 2002.

BORBA, M. C; PENTEADO, M. G. Informática e Educação Matemática. 3. ed. Belo Horizonte: Autêntica, 2003.

BORGES, I. Prática pedagógica, processos interativos humanos e a construção do conhecimento usando a internet: uma análise a partir da teoria histórico-cultural de Lev S. Vygotsky. Dissertação (Mestrado em Engenharia de Produção) - Departamento de Engenharia de Produção e Sistemas, Universidade Federal de Santa Catarina, Florianópolis, SC, 2000.

CAMPOS, P. K. A formação docente integrada ao ambiente computacional e sua (re)significação na prática pedagógica em matemática: análise de um caso. Dissertação (Mestrado em Educação) — Universidade São Francisco, Itatiba, 2007. 141 p.

DAVÝDOV, V. V. Tipos de generalización en la enseñanza. Havana: Pueblo y Educacion, 1982.

DAVÝDOV, V. V. La enseñanza escolar y el desarrollo psíquico. Moscu: Editorial Progreso, 1988.

DIAS, M. S.; COBIANCHI, A. S. Correlação do lógico e do histórico no ensino dos números reais. In: Encontro paulista de educação matemática, 7., 2004, São Paulo. Anais... São Paulo: SBEM, 2004. 11 p.

FIORENTINI, D; CASTRO, F C. Tornando-se professor de Matemática: o caso de Allan em Prática de Ensino e Estágio Supervisionado. In: Fiorentini, Dario. (Org.) Formação de professores de Matemática: explorando novos caminhos com outros olhares. Campinas, SP: Mercado de Letras, 2003. p.121-156.

FREITAS, M. T. M. A escrita no processo de formação contínua do professor de Matemática. Tese (Doutorado em Educação: Educação Matemática) - Faculdade de Educação, Universidade Estadual de Campinas, Campinas, 2006. 299p. 
GIARDINETTO, J. R. B. Matemática escolar e matemática da vida cotidiana. Campinas: Autores Associados, 1999.

GRAVINA, M. A, SANTAROSA, L. M. C. A Aprendizagem da Matemática em Ambientes Informatizados. Informática na Educação: Teoria e Prática.1998. vol. 1, n. 1. Porto Alegre: UFRGS - Curso de Pós-Graduação em Informática na Educação.

LANNER DE MOURA, A R; MISKULIN, R. G. S; MELO, G. F. A. A tecnologia computacional como potencializadora da aprendizagem 202 compartilhada do conceito matemático. In: MATOS, João Filipe; FERNANDES, Elsa. (Org.). Investigação em educação matemática: perspectivas e problemas. Lisboa: Associação de Professores de Matemática, 2000. p.145-152.

LEONTIEV, A. N. O desenvolvimento do psiquismo. Lisboa: Livros Horizonte, 1978.

LEONTIEV, A. N. Actividad, conciencia, personalidad. La Habana: Editorial Pueblo y Educación, 1983. $2^{a}$ reimpressão.

LESSARD, C; TARDIF, M. As transformações atuais do ensino: três cenários possíveis na evolução da profissão professor? In: TARDIF, Maurice; LESSARD, Claude (Org). $\mathbf{O}$ ofício de professor: história, perspectivas e desafios internacionais. Tradução Lucy Magalhães. Petrópolis: Vozes, 2008.

LIMA, L. C. A fração: repartindo o universo. São Paulo: CETEAC, 1998.

MARCO, F. F. Atividades computacionais de ensino na formação inicial do professor de matemática. Tese (Doutorado em Educação) - Universidade Estadual de CampinasCampinas, 2009.

MELLO, G. N. Formação inicial de professores para a educação básica: uma (re) visão radical. São Paulo em perspectiva, v. 14, n. 1, p. 98-110, 2000.

MISKULIN, R. G. S; LANNER DE MOURA, A. R; SILVA, M. R. Um estudo sobre a dimensão semiótica da tecnologia na educação e na educação matemática. In: Seminário Internacional De Pesquisa Em Educação Matemática, 2., out./nov. 2003, Santos. Anais..., 2003. Santos, SP. (Versão em CD-ROM).

MORETTI, V. D.; MOURA, M. O. de. A formação docente na perspectiva históricocultural: em busca da superação da competência individual. Rev. psicol. polít., São Paulo , v. 10, n. 20, p. 345-361, dez. $2010 \quad$. Disponível em $<$ http://pepsic.bvsalud.org/scielo.php?script=sci_arttext\&pid=S1519-

549X2010000200012\&lng=pt\&nrm=iso>. acessos em 02 fev. 2018.

MOURA, M. O. Pesquisa colaborativa: um foco na ação formadora. In: BARBOSA, Raquel Lazzari Leite (Org.) Trajetórias e perspectivas da formação de educadores. São Paulo: Editora Unesp, 2004. Cap.18, p. 257-284.

MOURA, M. O. de; SFORNI, M. S. F. de; ARAUJO, E. S. Objetivação e apropriação de 
conhecimentos na atividade orientadora de ensino. Teoria e Prática da Educação, Maringá, v. 14 , n. 1, p. 39-50, 2011.

NIESS, M. L. et al. (2009). Mathematics Teacher TPACK Standards and Development Model. Contemporary Issues in Technology and Teacher Education, v., n. 1

OLIVEIRA, M. K. Vygotsky: aprendizado e desenvolvimento: um processo sóciohistórico. 4. ed. São Paulo: Scipione, 1997.

PAPERT, S. A máquina das crianças: repensando a escola na era da informática. Tradução Sandra Costa. Porto Alegre: Artes Médicas, 1994.

ROSA, J. E. Proposições de Davydov para o ensino de Matemática no primeiro ano escolar: Inter-relações dos sistemas de sistema de significações numéricas. 2012. 244 f. Tese (Doutorado em Educação)-Universidade Federal do Paraná, Curitiba, 2012.

RODRIGUES, A; SOUZA JÚNIOR, A. J; LOPES, C. R. Objeto de aprendizagem na escola pública. In: LOPES, Carlos Roberto; FERNANDES, Márcia Aparecida. (Org.). Informática na educação: elaboração de objetos de aprendizagem. Uberlândia: EDUFU, 2007.

SANTAELLA, L. Cultura das mídias. São Paulo: Experimento, 1996. 292 p.

TAJRA, S. F. Informática na Educação: novas ferramentas pedagógicas para o professor na atualidade. Sanmya Feitosa Tajra. 3.ed. rev. atual e ampl. - São Paulo: Érica, 2001

VALENTE, J. A; ALMEIDA, F. J. Visão analítica da informática na educação no Brasil: a questão da formação do professor. Revista Brasileira de Informática na Educação Sociedade Brasileira de Informática na Educação, n.1, pp.45-60, 1997.

VALENTE, J. A. (Org.). Computadores e conhecimento: repensando a educação. 2. ed. Campinas: UNICAMP/NIED, 1998.

VYGOTSKY, L. S. A construção do pensamento e da linguagem. São Paulo: Martins Fontes, 2001.

VYGOTSKI, L. S. Obras escogidas. Tomo III. Madrid: Visor Distribuidores, S.A., 1995.

VYGOTSKI, L. S. Pensamento e Linguagem. 3 ed. São Paulo: Martins Fontes, 2005.

WILEY, D. A. Connecting learning objects to instructional design theory: A definition, a metaphor, and a taxonomy. In: The Instructional Use of Learning Objects, Bloomington: Agency for Instructional Technology and Association for Educational Communications and Technology. 